\title{
Stem Cell Health and Tissue Regeneration in Microgravity
}

\author{
Elizabeth Blaber, Kevin Sato, and Eduardo A.C. Almeida*
}

\section{ABSTRACT}

Exposure to microgravity causes significant mechanical unloading of mammalian tissues, resulting in rapid alterations of their physiology, which poses a significant risk for long-duration manned spaceflight. The immediate degenerative effects of spaceflight we understand best are those studied during short-term low-Earth-orbit experiments, and include rapid microgravityadaptive bone and muscle loss, loss of cardiovascular capacity, defects in wound and bone fracture healing, and impaired immune function. Over the long-term, exposure to microgravity may cause severe deficits in mammalian stem cell-based tissue regenerative health, including, osteogenesis, hematopoiesis, and lymphopoeisis, as well as cause significant stem cellbased tissue degeneration in amphibian tail and lens regeneration. To address the needs for stem cell and other cell science research on the International Space Station (ISS), NASA has developed the new Bioculture System that will allow investigators to initiate and conduct on-orbit experiments that astronauts will be able to monitor and interact with during the course of cell cultures. This cell culture capability combined with advanced technologies for molecular biology and on-orbit measurement of gene expression (WetLab2) and other tools that are now coming online bring the ISS National Laboratory a step closer to becoming a fully functional space laboratory for advancing space biological sciences.

\section{INTRODUCTION}

Humanity has both a strong aspiration and a practical need to become a space-faring race, and to expand and establish its presence on other solar system moons and planets, such as Mars. However, in order to achieve these long-term aims, terrestrial life, both human and other species essential to our survival, need to overcome the many challenges that arise from

Space Biosciences Division, NASA Ames Research Center, Moffett Field, California.

*(Correspondence: e.almeida@nasa.gov) existing in a low-gravity space environment. The predominant space conditions that differ from those found on Earth are the absence of gravity, absence of a charged-particle-deflecting geomagnetic field, and the lack of an atmosphere. Because of this unique environment, we need to understand the response of terrestrial life to space from single cells to the level of integrated tissues and organ systems, before further human space exploration and expansion beyond Earth can occur. Here we focus on how the lack of gravity mechanostimulatory forces in space is a major concern for stem cell and tissue regenerative health during long-term spaceflight, and how NASA ISS Space Biology experimental capabilities can address these science questions.

\section{The space environment induces tissue degeneration}

Astronauts in space are exposed to several different gravitational environments that affect basic biological processes. The majority of human spaceflights have been conducted in low Earth orbit (LEO), which 
is approximately $160-2,000 \mathrm{~km}$ above the Earth's surface [1], still protected from charged particle space radiation. Normal terrestrial gravity $(1 \mathrm{~g})$ forces objects to accelerate toward the center of the Earth. The Earth's surface resists the downward acceleration of gravity, and it is this force that shapes the nature of our musculoskeletal system and how it supports our body. Therefore, on Earth, organisms are constantly subjected to contact forces that provide an array of mechanical stimulation essential for the function of many physiological systems. The influence of these mechanical contact forces on the human body is especially evident in the effects of physical exercise loads on the weight-bearing skeleton. Increased load during weight-bearing exercise causes increased musculoskeletal growth to enable the body to withstand these increased forces. For orbital flight around the Earth, the force of the Earth's gravity keeps the spacecraft moving in an orbital path. Although zero gravity is not experienced in LEO, near-zero contact forces are exerted by the spacecraft on its inhabitants, or in other words, the astronauts and the contents of the spacecraft are in a state of free fall in orbit around the Earth [1], resulting in gravitational force of approximately $1 \times 10^{-6} \mathrm{~g}$, microgravity. In space, this lack of normal gravity and resulting loss of mechanical stimulation of cells and tissues are responsible for many of the physiological problems that astronauts experience-from space motion sickness and otolith dysfunction, to cardiovascular, bone, and muscle degeneration. Specifically, as astronauts have journeyed in microgravity, the spaceflight gravitational environment revealed many immediate tissue degenerative consequences for life. These include bone loss [2-9], muscle loss [2,10-15], loss of cardiovascular capacity [16-19], possible defects in wound [20-22] and bone fracture healing [23-25], and impaired immune function [6,26-31]. The majority of space biological experimentation was conducted on the Space Shuttle, which supported short-term (1-2 weeks) experiments on spaceflight effects. In particular, research concentrated on the more noticeable changes that occur as an adaptation to moving from loaded conditions at $1 \mathrm{~g}$ to unloaded conditions in microgravity. In the long-term, however, microgravity may also affect normal ongoing tissue regenerative growth and repair, a process dependent on the proliferation and differentiation of tissuespecific adult stem cells that are progenitors of mature terminally differentiated cells in most tissues. New investigations on ISS are now focusing on longer-term experiments that are becoming possible with ISS completion and ISS-resident experimental biology hardware.

\section{The physiological effects of spaceflight on stem cells}

The cell culture experiments conducted in space have used a variety of cell types, including somatic stem cells, embryonic stem cells, and cell culture lines, to attempt to determine the influence of microgravity on cellular function (Fig. 1). Through these experiments, the detrimental effects of microgravity on cellular function were consistently revealed, including inhibition of osteoblast differentiation $[2,32,33]$, reduced osteoblast numbers [34], atrophy of skeletal muscle cells $[14,35,36]$, impaired activation of immune cells and consequently the immune system [37-39], abnormal formation of chondrocytes [40], and collapse of the cytoskeleton in $\mathrm{T}$ lymphoblastoid cells (Jurkat) [41], thereby displaying the impact of microgravity on various physiological systems.

In a majority of cases, microgravity cell studies focused on terminally differentiated cells and immortalized cell lines. However, considering the widespread effects of mechanical unloading on the body, it is likely that somatic stem cells will also be adversely affected by this environment. Somatic stem cells have the ability to differentiate into a number of lineage-specific terminally differentiated cells, as such altered somatic stem cell function will have significant ramifications to multiple cell lineages throughout the body. A very small subset of spaceflight experiments have focused on somatic stem cells, with the majority studying the differentiation and proliferation capacity of bone marrow-derived hematopoietic stem cells (HSCs) and mesenchymal stem cells. Exposure to spaceflight following addition of osteogenic differentiation factors resulted in increased expression of genes related to neural development, neural morphogenesis, and transmission of nerve impulses and synapses in studies conducted on ISS [42]. This same study found increased expression of cell cycle arrest molecules indicating either increased differentiation of cells in space or activation of cellular quiescence or senescence [42]. In the HSC lineage, a suppression of proliferation and differentiation has also been noted $[6,26,43]$. Additionally, shifts in immune cell phenotypes were noted in experiments flown on board STS-108 with an increase in the numbers of bone marrow-derived $\mathrm{T}$ cells and a decrease in bone marrowderived B cells [44]. Studies on STS-63 and STS-69 with CD34+ bone marrow progenitor cells revealed decreases in total cell number in microgravity samples, and additionally decreased erythropoiesis and increased macrophage differentiation [45]. Furthermore, studies investigating the bone marrow stem cell population in the femoral head of space-flown mice revealed an accumulation of red blood cells, decreased numbers of megakaryocytes, and decreased capacity of both mesenchymal and hematopoietic stem cells to differentiate into terminal lineages [43]. Such results highlight the alterations in stem cell proliferation and differentiation during spaceflight.

Recent spaceflight experiments addressed the role of microgravity in regulating stem cell-based tissue regeneration. In collaborative NASA-U.S./Russian experiments conducted during the Foton M2 and M3 missions, tissue regeneration in microgravity was studied using a lower vertebrate newt tail blastema model, which established that microgravity inhibited the transition between stem cell 
FIG. 1. The experimental models used to study stem cells in space include the regenerating tail in the newt (Pleurodeles waltl) (A), which contains rapidly proliferating blastema-derived tissue-specific de-differentiated stem cells shown here labeled in red with BrdU (B); mouse embryonic stem cells (C), differentiated into embryoid bodies during spaceflight $(D)$; and mouse bone marrow $(E)$, where hematopoietic $(F)$ and mesenchymal stem cell lineage differentiation have been investigated using cell and molecular approaches.
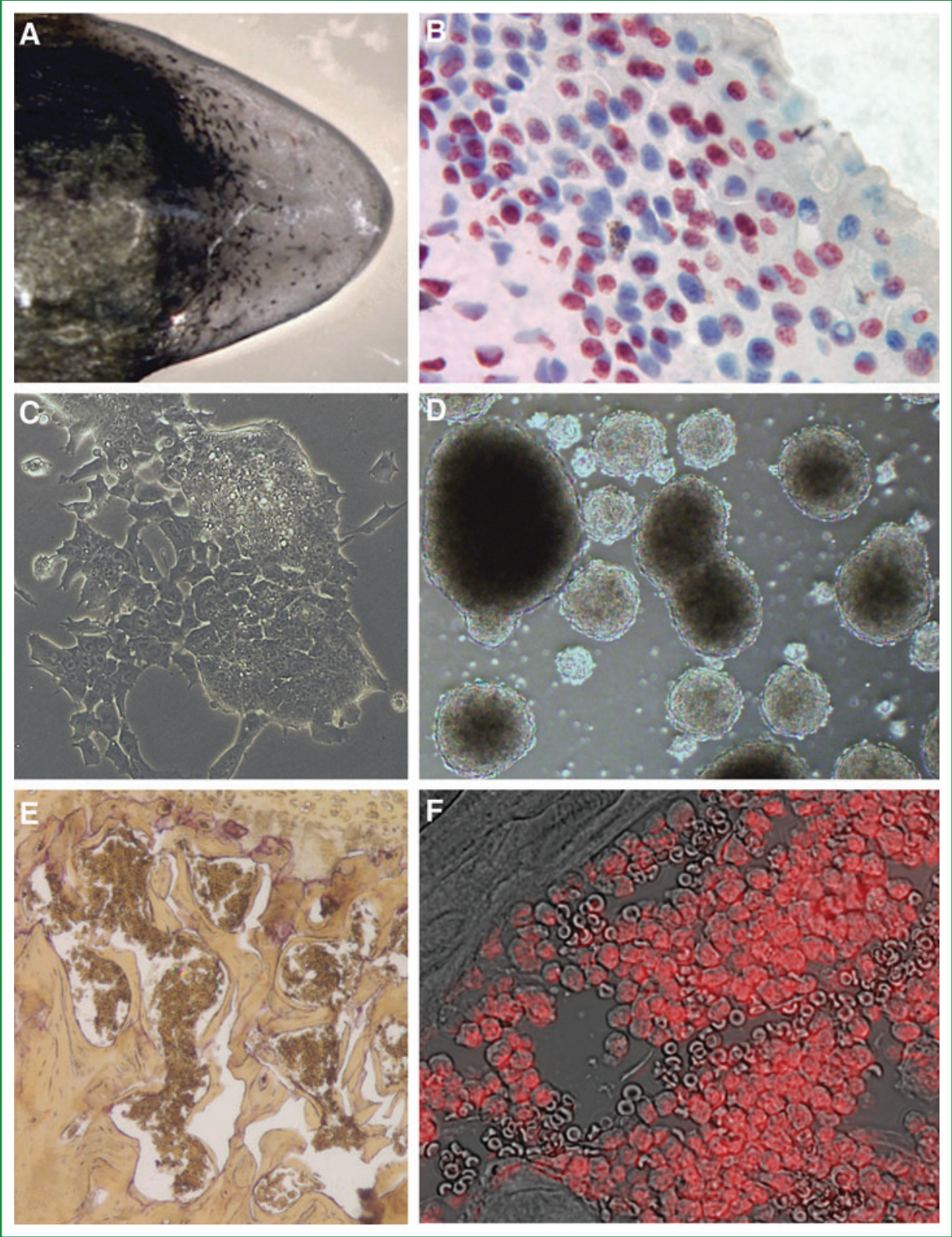

progenitor and differentiated cells. Conversely, ground centrifugation experiments showed that hypergravity promoted stem cell-based tissue regeneration by stimulating progenitor differentiation. In the STS-131 and STS135 space shuttle missions to ISS, the influence of microgravity on embryonic stem cell differentiation was studied by inducing differentiation into an early embryogenesis model, of embryoid body formation, and with the terminal differentiation of the keratinocyte lineage. In both studies, microgravity significantly impaired the ability of embryonic stem cells to express differentiation markers and instead resulted in maintenance of "stemness" [46-48]. Similar results were seen in studies of somatic bone marrow stem cells isolated from mouse bones flown on the STS-131 mission. In these studies, microgravity caused an inhibition of differentiation in mouse bone marrow osteoprogenitors required for normal bone regeneration, accompanied by $\mathrm{p} 21$ mediated cell cycle arrest $[43,49]$. Upon reloading following microgravity, the regenerative potential of bone marrow stromal cells to differentiate was greatly increased reflecting an accumulation of undifferentiated stem cell precursors in bone marrow. Studies of gene expression in these cells displayed widespread downregulation of hematopoietic and mesenchymal stem cell marker genes, with no alteration in early markers of stemness. Recent NASA-U.S./Russian microgravity experiments in the Bion M1 mission confirm previous results on stem cell differentiation inhibition by microgravity, and accumulation of stem cell precursors in bone marrow (E. Almeida, unpublished data).

In total, these recent experiments suggest a possible universal microgravity response by stem cells across various cell, tissue, and organismal experimental models, consisting of a partial inhibition of the transition from dividing progenitors to cell cycle-arrested, terminally differentiated adult cells. Such a fundamental need for gravity mechanical stimulation to maintain stem cell regenerative health appears more and more to be a basic feature of mammalian life on Earth at $1 \mathrm{~g}$, and it suggests that long-term spaceflight in microgravity may cause a broad inhibition of stem cell-based tissue regeneration.

\section{New capability for}

\section{conducting stem cell \\ culture studies on ISS}

The Cell Culture Module (CCM; DoD) used in many of the experiments described 
above was designed in the 1990s for the Space Shuttle middeck locker, and it required functional and operational upgrades for long-duration ISS experiments. The CCM design supported fully automated hollow fiber bioreactor cell culturing for up to 2 weeks with fixation on-orbit or live cell return, but it had no options to start cultures on-orbit, collect samples of the cultures, conduct microscopic observations, change the culture medium, and conduct other standard cell culture activities on-orbit. As the NASA Space Life and Physical Sciences and ISS programs sought to replace the CCM hardware for the ISS National Lab, it incorporated requirements for extensive user-interactive features to allow on-orbit cell science experimentation in the new Bioculture System (Fig. 2). Specifically, the new Bioculture System is both an automated and crew-accessible cell culture hardware that provides controlled maintenance/growth medium, gas, and temperature of cell cultures in the microgravity environment of space. The system incorporates 10 independent cassettes each with perfusion-based cell culture porous-hollow fiber bioreactors. The porous hollow fibers protect cells from fluid shear forces created by perfused feeding, which would otherwise mask microgravity effects. Computer-controlled fluidics allow for manual or fully automated operation of medium change, injections, medium or cell sample collection, and an internal refrigerated storage compartment. NASA envisions investigators being able to conduct cell science experiments end-to-end on ISS, with on-orbit capabilities for initiating cultures from frozen stocks, sampling cell cultures for on-orbit studies of gene expression by qPCR (using the WetLab2 capabilities), and for microscopic observation and imaging, adding growth and differentiation factors to cultures depending on culture progression, and preserving and/or isolating RNA, DNA, and proteins on-orbit. This approach of using ISS as a National Laboratory, similar to ground-based laboratories, is challenging but also promises improved science outcomes. Furthermore, it allows

FIG. 2. The NASA Bioculture System consists of a docking station housing and supporting 10 independent cell culture cassettes, (A) designed for the ISS express rack. Each durable cassette contains an internal disposable flow path (B) consisting of media bags, a hollow fiber bioreactor, and fluidic components, including tubing, pumps, and valves. The bioreactor (C) is uniquely capable of delivering nutrients and removing waste through diffusion without direct mechanostimulatory fluid flow in contact with the cells, and supports cell adhesion to fibers or to matrix-coated alginate microcarrier beads such as in nonproliferating human induced pluripotent stem cells-derived cardiomyocytes (D), or proliferating osteocytic cultures (E).

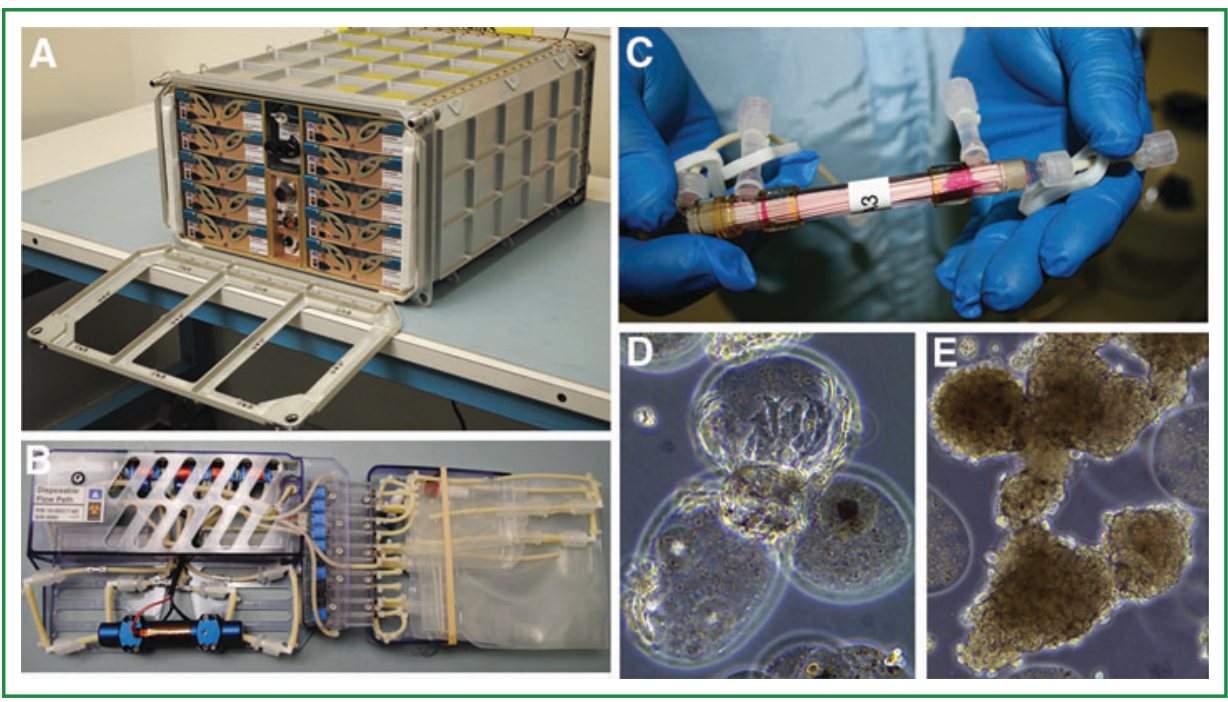

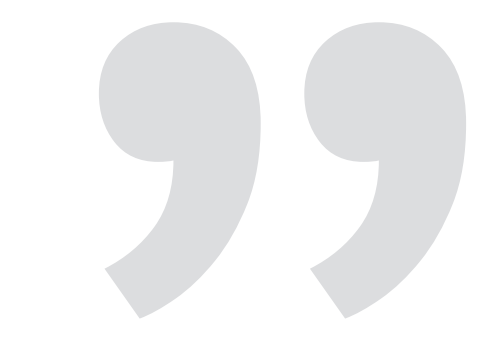

\section{It allows NASA to gain greater insight into how the space environment affects stem cells in microgravity.}

NASA to develop the capability to conduct biological experimentation in space for future long-term exploration, and to gain greater insight into how the space environment affects stem cells and stem cell-based regenerative health in microgravity.

\section{ACKNOWLEDGMENTS}

This work was supported by the NASA Space Biology Program, NASA Fundamental Space Biology Grants NNH08ZTT003N and NNH07ZTT001N to E. Almeida, and NASA Postdoctoral Program Fellowship to E. Blaber.

\section{Author Disclosure Statement}

No competing financial interests exist.

\section{REFERENCES}

1. Lackner JR and P DiZio. (2000). Human orientation and movement control in weightless and artificial gravity environments. Exp Brain Res 130:2-26.

2. Nabavi N, et al. (2011). Effects of microgravity on osteoclast bone resorption and osteoblast cytoskeletal organization and adhesion. Bone 49:965-974. 
3. Hughes-Fulford M. (2003). Function of the cytoskeleton in gravisensing during spaceflight. Adv Space Res 32:1585-1593.

4. Ohshima H. (2006). [Bone loss and bone metabolism in astronauts during long-duration space flight]. Clin Calcium 16:81-85.

5. Smith SM and M Heer. (2002). Calcium and bone metabolism during space flight. Nutrition 18:849-852.

6. Zayzafoon M, VE Meyers and JM McDonald. (2005). Microgravity: the immune response and bone. Immunol Rev 208:267-280.

7. Zerath E. (1998). Effects of microgravity on bone and calcium homeostasis. Adv Space Res 21:1049-1058.

8. Tamma R, et al. (2009). Microgravity during spaceflight directly affects in vitro osteoclastogenesis and bone resorption. FASEB J 23:2549-2554.

9. Bikle DD, T Sakata and BP Halloran. (2003). The impact of skeletal unloading on bone formation. Gravit Space Biol Bull 16:45-54.

10. Bajotto G and Y Shimomura. (2006). Determinants of disuse-induced skeletal muscle atrophy: exercise and nutrition countermeasures to prevent protein loss. J Nutr Sci Vitaminol (Tokyo) 52:233247.

11. Ferrando AA, D Paddon-Jones and RR Wolfe. (2002). Alterations in protein metabolism during space flight and inactivity. Nutrition 18:837-841.

12. Fitts RH, DR Riley and JJ Widrick. (2001). Functional and structural adaptations of skeletal muscle to microgravity. J Exp Biol 204:3201-3208.

13. Ogneva IV. (2010). Transversal stiffness of fibers and desmin content in leg muscles of rats under gravitational unloading of various durations. J Appl Physiol 109:1702-1709.

14. Fitts RH, et al. (2010). Prolonged space flight-induced alterations in the structure and function of human skeletal muscle fibres. J Physiol 588:3567-3592.

15. Trappe S, et al. (2009). Exercise in space: human skeletal muscle after 6 months aboard the International Space Station. J Appl Physiol 106:1159-1168. 16. Aubert AE, $F$ Beckers and $B$ Verheyden. (2005). Cardiovascular function and basics of physiology in microgravity. Acta Cardiol 60:129-151. 17. Convertino VA. (2005). Consequences of cardiovascular adaptation to spaceflight: implications for the use of pharmacological countermeasures. Gravit Space Biol Bull 18:59-69.

18. Cooke WH and VA Convertino. (2005). Cardiovascular consequences of weightlessness promote advances in clinical and trauma care. Curr Pharm Biotechnol 6:285-297.

19. Norsk P. (2005). Cardiovascular and fluid volume control in humans in space. Curr Pharm Biotechnol 6:325-330.

20. Kirkpatrick AW, et al. (1997). Blunt trauma and operative care in microgravity: a review of microgravity physiology and surgical investigations with implications for critical care and operative treatment in space. J Am Coll Surg 184:441-453.

21. Radek KA, et al. (2008). Mechanical unloading impairs keratinocyte migration and angiogenesis during cutaneous wound healing. J Appl Physiol 104:1295-1303.

22. Martinez DA, et al. (2007). Temporal extracellular matrix adaptations in ligament during wound healing and hindlimb unloading. Am J Physiol Regul Integr Comp Physiol 293:R1552R1560.

23. Durnova GN, et al. (1991). [The effect of weightlessness on fracture healing of rats flown on the biosatellite Cosmos-2044]. Kosm Biol Aviakosm Med 25:29-33.

24. Kaplansky AS, et al. (1991). The effect of microgravity on bone fracture healing in rats flown on Cosmos-2044. Physiologist 34:S196-S199.

25. Kirchen ME, et al. (1995). Effects of microgravity on bone healing in a rat fibular osteotomy model. Clin Orthop Relat Res 1995:231-242.

26. Borchers AT, CL Keen and ME Gershwin. (2002). Microgravity and immune responsiveness: implications for space travel. Nutrition 18:889-898.

27. Klaus DM and HN Howard. (2006). Antibiotic efficacy and microbial virulence during space flight. Trends Biotechnol 24:131-136.

28. Sonnenfeld G. (2005). The immune system in space, including Earth-based benefits of space-based research. Curr Pharm Biotechnol 6:343-349.
29. Crucian B, et al. (2011). Monocyte phenotype and cytokine production profiles are dysregulated by short-duration spaceflight. Aviat Space Environ Med 82:857-862.

30. Stowe RP, CF Sams and DL Pierson. (2011). Adrenocortical and immune responses following short- and longduration spaceflight. Aviat Space Environ Med 82:627-634.

31. Sonnenfeld G. (2005). Use of animal models for space flight physiology studies, with special focus on the immune system. Gravit Space Biol Bull 18:31-35.

32. Landis W, et al. (2000). Spaceflight effects on cultured embryonic chick bone cells. J Bone Min Res 15:1099-1112.

33. Carmeliet G, G. Nys and R Bouillon. (1997). Microgravity reduces the differentiation of human osteoblastic MG63 cells. J Bone Miner Res 12:786-794.

34. Hughes-Fulford M and ML Lewis. (1996). Effects of microgravity on osteoblast growth activation. Exp Cell Res 224:103-109.

35. Vandenberg H, et al. (1999). Space travel directly induces skeletal muscle atrophy. FASEB J 13:1031-1038.

36. Allen DL, et al. (2009). Effects of spaceflight on murine skeletal muscle gene expression. J Appl Physiol 106:582-595.

37. Cogoli A. (1996). Gravitational physiology of human immune cells: a review of in vivo, ex vivo and in vitro studies. J Grav Physiol 3:1-10.

38. Gridley DS, et al. (2009). Spaceflight effects on $\mathrm{T}$ lymphocyte distribution, function and gene expression. J Appl Physiol 106:194-202.

39. Buravkova LB, et al. (2008). Cytotoxic activity of natural killer cells in vitro under microgravity. Dokl Biol Sci 421:275-277.

40. Freed L, et al. (1997). Tissue engineering of cartilage in space. Proc Natl Acad Sci USA 94:13885-13890.

41. Lewis ML, et al. (1998). Spaceflight alters microtubules and increases apoptosis in human lymphocytes (Jurkat). FASEB J 12:1007-1018.

42. Monticone M, et al. (2010). Activation of nervous system development genes in bone marrow derived mesenchymal stem cells following spaceflight exposure. J Cell Biochem 111:442-452. 
43. Blaber EA, et al. (2014). Mechanical unloading of bone in microgravity reduces mesenchymal and hematopoietic stem cell-mediated tissue regeneration. Stem Cell Res 13:181-201.

44. Pecaut MJ, et al. (2003). Genetic models in applied physiology: selected contribution: effects of spaceflight on immunity in the C57BL/6 mouse. I. Immune population distributions. J Appl Physiol 94:2085-2094.

45. Davis TA, et al. (1996). Effect of spaceflight on human stem cell hematopoiesis: suppression of erythropoiesis and myelopoiesis. J Leukoc Biol 60:69-76.

46. Blaber E, H Finkelstein, N Dvorochkin, BP Burns and EA Almeida. (2011). Spaceflight alters the migratory ability of stem cell derived keratinocytes resulting in decreased wound healing potential. Mol Biol Cell 22:4705 (Abstract \#1533).

47. Finkelstein H, N Dvorochkin, R Yousuf, RK Globus and EA Almeida. (2010). Spaceflight reduces the tissue regenerative potential of stem cells by decreaseing proliferation and increasing early differentiation. Gravit Space Biol 24:38. 48. Finkelstein $\mathrm{H}, \mathrm{E}$ Blaber, N Dvorochkin, RK Globus, BP Burns and EA Almeida. (2011). Spaceflight reduces the wound healing potential of stem cell derived keratinocytes by decreasing migration. Gravit Space Biol 26(1).

49. Blaber EA, et al. (2013). Microgravity induces pelvic bone loss through osteoclastic activity, osteocytic osteolysis, and osteoblastic cell cycle inhibition by CDKN1a/p21. PLoS One 8:e61372. 